\title{
Correlation between site occupancies and spin-glass transition in skyrmion host $\mathrm{Co}_{10-\frac{x}{2}} \mathbf{Z n}_{10-\frac{x}{2}} \mathbf{M n}_{x}$
}

\author{
T. Nakajima, ${ }^{1,2, *}$ K. Karube, ${ }^{2}$ Y. Ishikawa, ${ }^{3}$ M. Yonemura, ${ }^{3}$ N. Reynolds,${ }^{4,5}$ J. S. White, ${ }^{4}$ H. M. Rønnow, ${ }^{5}$ A. Kikkawa, ${ }^{2}$ \\ Y. Tokunaga, ${ }^{2,6}$ Y. Taguchi, ${ }^{2}$ Y. Tokura, ${ }^{1,2}$ and T. Arima ${ }^{2,6}$ \\ ${ }^{1}$ Department of Applied Physics and Quantum Phase Electronics Center, University of Tokyo, Tokyo 113-8656, Japan \\ ${ }^{2}$ RIKEN Center for Emergent Matter Science, Saitama 351-0198, Japan \\ ${ }^{3}$ Institute of Materials Structure Science, KEK, Tokai, Ibaraki 319-1106, Japan \\ ${ }^{4}$ Laboratory for Neutron Scattering and Imaging, Paul Scherrer Institute, CH-5232 Villigen, Switzerland \\ ${ }^{5}$ Laboratory for Quantum Magnetism, Institute of Physics, École Polytechnique Fédérale de Lausanne, CH-1015 Lausanne, Switzerland \\ ${ }^{6}$ Department of Advanced Materials Science, University of Tokyo, Kashiwa 277-8561, Japan
}

(Received 15 May 2019; published 16 August 2019)

\begin{abstract}
Ternary alloys Co-Zn-Mn with $\beta$-Mn-type structure exhibit helimagnetic order and skyrmion lattice states in a low-Mn-concentration region. Recent studies on $\mathrm{Co}_{10-\frac{x}{2}} \mathrm{Zn}_{10-\frac{x}{2}} \mathrm{Mn}_{x}(\mathrm{CZM})$ revealed that the magnetic modulation period and the paramagnetic-to-helimagnetic transition temperature are reduced with increasing Mn concentration $x$ [Tokunaga et al., Nat. Commun. 6, 7638 (2015)] and that the helimagnetic order is replaced with a spin-glass state in a high- $x$ region [Karube et al., Sci. Adv. 4, eaar7043 (2018)]. To understand the microscopic mechanism of the Mn substitution effect, we have performed crystal structure analyses of a series of CZM samples with $x=4,5,6,7,8,10,12$, and 16 by neutron powder diffraction. We present a model for the site occupancies of $\mathrm{Co}, \mathrm{Zn}$, and $\mathrm{Mn}$ in the unit cell, which has two independent crystallographic sites, specifically $8 c$ and $12 d$ sites. $\mathrm{Zn}$ atoms are always accommodated in the $12 d$ sites, and Co atoms prefer the $8 c$ sites, although some of them also exist in the $12 d$ sites in a low- $x$ region. Mn atoms mainly prefer the $12 d$ sites but also share the $8 c$ site with Co atoms in the region of $x \geqslant 4$. A comparison between the site occupancies and the $x-T$ magnetic phase diagram suggests that the amount of $\mathrm{Mn}$ in the $8 c$ sites is relevant to the emergence of the spin-glass state.
\end{abstract}

DOI: 10.1103/PhysRevB.100.064407

\section{INTRODUCTION}

Magnetic skyrmions are nanometer-scale magnetic vortices with topologically nontrivial spin textures [1-3]. They were discovered in the binary magnetic compound $\mathrm{MnSi}$, which has a chiral crystal structure belonging to the space group of $P 2{ }_{1} 3$. This compound is known to exhibit a longperiod modulated helimagnetic phase in zero magnetic field owing to a combination of ferromagnetic exchange interactions, $J S_{i} \cdot S_{j}$, and Dzyaloshinskii-Moriya (DM) interactions, $D \cdot\left(S_{i} \times S_{j}\right)$. By applying a moderate magnetic field just below the critical temperature, a triangular skyrmion lattice (SkL) is induced. The discovery of the SkL phase in $\mathrm{MnSi}$ stimulated not only extensive exploration of new skyrmion host compounds, such as (Fe,Co)Si [4], FeGe [5], and $\mathrm{Cu}_{2} \mathrm{OSeO}_{3}$ [6], but also proposals and attempts to develop skyrmion-based spintronic devices, in which each skyrmion acts as an information carrier [7-10]. For the technological applications, it is important to develop methodologies to control the working temperature and the size of skyrmions; they are mainly determined by the magnitude of $J$ and the ratio of $D$ to $J$, respectively. Recent studies on metallic skyrmionhost materials revealed that the sign and magnitude of $D$ are determined not only by the local spin-orbit coupling on the magnetic atoms as in the case of localized spin systems but also by the band filling [11-15].

*taro.nakajima@ap.t.u-tokyo.ac.jp
Ternary alloys $\mathrm{Co}_{10-\frac{x}{2}} \mathrm{Zn}_{10-\frac{x}{2}} \mathrm{Mn}_{x}(\mathrm{CZM})$ are skyrmionhost materials with $\beta$-Mn-type chiral cubic crystal structure belonging to the space group of $P 4_{1} 32$ or $P 4_{3} 32$ [16-19]. An end compound $\mathrm{Co}_{10} \mathrm{Zn}_{10}$ shows long-period helimagnetic order below $T_{C}=460 \mathrm{~K}$ (and a SkL phase as well in a magnetic field [20]), which indicates the existence of fairly large $J[16,21]$. A SkL phase was first reported in $\mathrm{Co}_{8} \mathrm{Zn}_{8} \mathrm{Mn}_{4}$ [16]. It was also reported that the Mn substitution markedly reduces $T_{C}$ and the modulation period of the helimagnetic order $\lambda_{m}$, which determines the size of the skyrmions, suggesting that in the CZM family, the magnitude of $J$ is controllable by $\mathrm{Mn}$ concentration. In addition, Mn substitution induces a spin-glass phase (vide infra). To understand the origins of the magnetism and related physical properties of this system, the understanding of the detailed crystal structure including the site occupancies of the respective magnetic ions and the resultant magnetic interactions are indispensable. In this paper, we address these issues by investigating crystal structures of a series of CZM samples by means of neutron powder diffraction.

Before going into the details of the experiments, we present an overview of the relationship between the $x$ - $T$ magnetic phase diagram and crystallographic site occupancies of Co, $\mathrm{Zn}$, and $\mathrm{Mn}$ deduced from the present study. Figure 1(a) shows the $x$-T magnetic phase diagram cited from Ref. [19]. As mentioned above, the Mn substitution reduces $T_{C}$. The helimagnetic phase disappears at around $x=7$, and the system exhibits a spin-glass state above the critical Mn concentration. Note that the spin-glass state also appears in a 


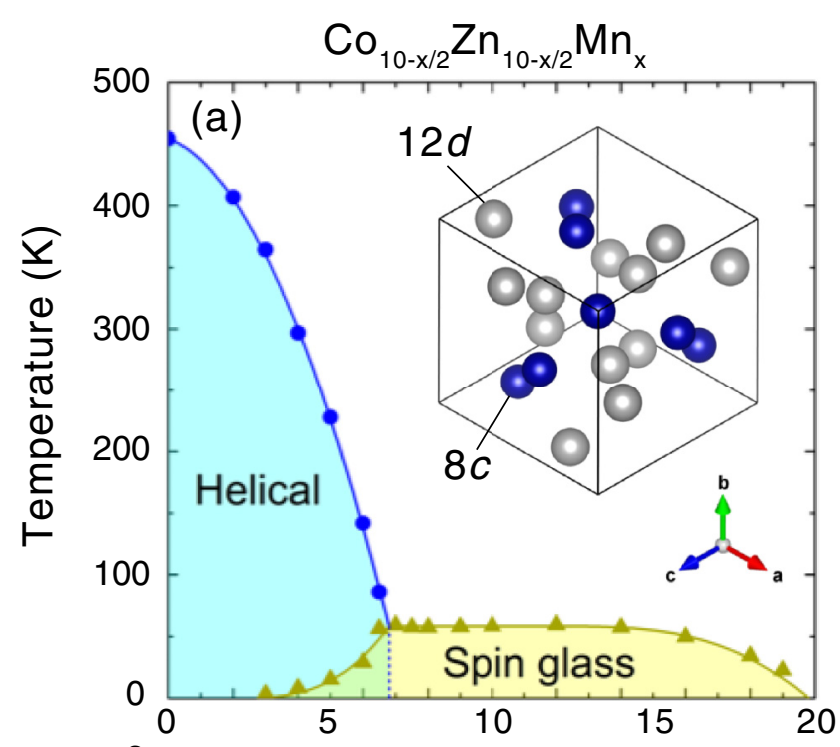

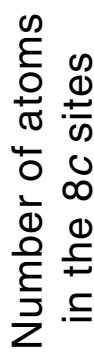
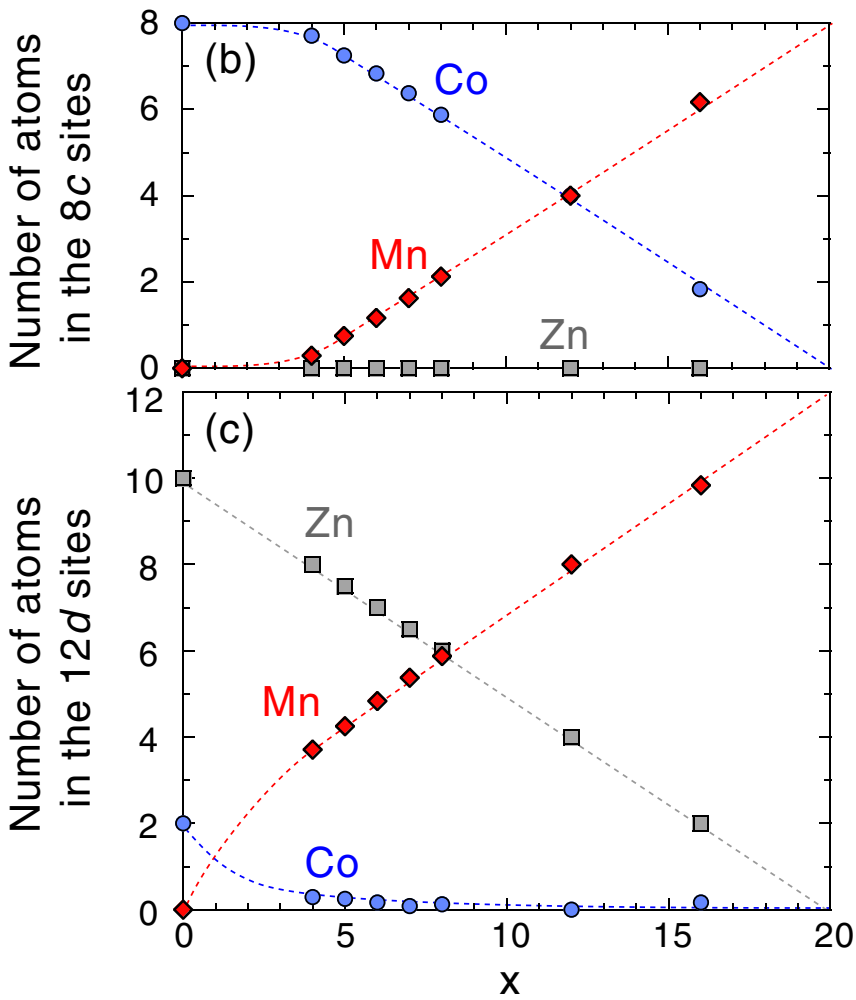

FIG. 1. (a) The $x-T$ magnetic phase diagram of $\mathrm{Co}_{10-\frac{x}{2}} \mathrm{Zn}_{10-\frac{x}{2}} \mathrm{Mn}_{x}$ cited from Ref. [19]. The inset shows the crystal structure and two independent sites. Site occupancies of Co, $\mathrm{Zn}$, and $\mathrm{Mn}$ for (b) $8 c$ and (c) $12 d$ sites deduced from the present study. The data points at $x=0$ are cited from Refs. [21,22]. Dotted lines are guides to the eyes.

Mn concentration range of $3 \leqslant x \leqslant 7$, where the system undergoes a reentrant transition to the spin-glass state while maintaining the helical order at low temperatures. The inset of Fig. 1(a) shows the crystal structure of CZM, which has two independent crystallographic sites, $8 c$ and $12 d$. The present structural refinements reveal that $\mathrm{Zn}$ atoms selectively occupy $12 d$ sites and that $8 c$ sites prefer Co atoms to $\mathrm{Mn}$ and $\mathrm{Zn}$. As $x$ increases, some Mn atoms start sharing the $8 c$ sites with Co, as shown in Figs. 1(b) and 1(c). We suggest that the number of $\mathrm{Mn}$ atoms in the $8 c$ sites is relevant to the emergence of the spin-glass state, implying that antiferromagnetic Mn-Mn spin correlation between neighboring $8 c$ sites leads to strong magnetic disorder in the system.

In the following, we present how we have drawn the conclusion mentioned above. It should be noted that it is impossible to determine the occupancies of three elements at two independent crystallographic sites. By combining structural and chemical analyses, we have experimentally determined the upper limits of Mn concentration for the two sites. Taking into account the site preference of $\mathrm{Co}$ and $\mathrm{Zn}$ reported in the previous study, we present a reasonable model for the site occupancies.

\section{PRELIMINARY DETAILS}

One of the most conventional techniques to determine crystal structures is X-ray diffraction (XRD). However, XRD is not suitable for the present study because $\mathrm{Co}, \mathrm{Zn}$, and $\mathrm{Mn}$ are very similar to each other in terms of number of electrons, which determines the atomic scattering factor for $\mathrm{x}$ rays. We used the neutron diffraction technique because the coherent nuclear scattering lengths of $\mathrm{Co}, \mathrm{Zn}$, and $\mathrm{Mn}$ atoms are largely different from each other, specifically, $b_{\mathrm{Co}}=2.49 \mathrm{fm}, b_{\mathrm{Zn}}=$ $5.68 \mathrm{fm}$, and $b_{\mathrm{Mn}}=-3.73 \mathrm{fm}$, respectively.

In neutron diffraction, the intensity of a nuclear Bragg reflection of an alloy is proportional to a square of the structure factor $F_{h k l}$, given by

$$
F_{h k l}=\sum_{j}\langle b\rangle_{j} e^{i \boldsymbol{d}_{j} \cdot \boldsymbol{\tau}_{h k l}},
$$

where the summation is taken for all the sites in a unit cell. $\langle b\rangle_{j}, d_{j}$, and $\tau_{h k l}$ are the weighted average of the scattering lengths, the fractional coordinates of the $j$ th site, and the reciprocal lattice vector for $h k l$, respectively. CZM has two independent crystallographic sites, $8 c$ and $12 d$, both of which may be randomly occupied by two or three elements. Accordingly, the structure factor is written as

$$
F_{h k l}=\sum_{j \in 8 c}\left\langle b_{8 c}\right\rangle e^{i \boldsymbol{d}_{j} \cdot \boldsymbol{\tau}_{h k l}}+\sum_{j \in 12 d}\left\langle b_{12 d}\right\rangle e^{i \boldsymbol{d}_{j} \cdot \boldsymbol{\tau}_{h k l}},
$$

where $\left\langle b_{8 c}\right\rangle$ and $\left\langle b_{12 d}\right\rangle$ are averaged scattering lengths for the $8 c$ and $12 d$ sites, respectively. In Rietveld analyses, these two parameters are refined to reproduce observed intensities of Bragg peaks. In practice, we also need a scale factor to compare absolute values of the observed intensities with the calculations. Therefore, one can determine only the ratio of $\left\langle b_{12 d}\right\rangle$ to $\left\langle b_{8 c}\right\rangle$. Here, we refer to the ratio as

$$
P=\frac{\left\langle b_{12 d}\right\rangle}{\left\langle b_{8 c}\right\rangle} \text {. }
$$

Obviously, the site occupancies of the three elements cannot be determined from the one experimental value. We thus need constraint equations to reduce the number of parameters.

$\left\langle b_{8 c}\right\rangle$ and $\left\langle b_{12 d}\right\rangle$ are given by

$$
\begin{gathered}
\left\langle b_{12 d}\right\rangle=X_{\mathrm{Co}} b_{\mathrm{Co}}+X_{\mathrm{Zn}} b_{\mathrm{Zn}}+X_{\mathrm{Mn}} b_{\mathrm{Mn}}, \\
\left\langle b_{8 c}\right\rangle=Y_{\mathrm{Co}} b_{\mathrm{Co}}+Y_{\mathrm{Zn}} b_{\mathrm{Zn}}+Y_{\mathrm{Mn}} b_{\mathrm{Mn}},
\end{gathered}
$$


where $X_{M}$ and $Y_{M}(M=\mathrm{Co}, \mathrm{Zn}$, or $\mathrm{Mn})$ are site occupancies for the element $M$ in the $12 d$ and $8 c$ sites, respectively. Here, we assume that either the $12 d$ or $8 c$ sites do not contain any vacancies. Thus,

$$
\begin{gathered}
X_{\mathrm{Co}}+X_{\mathrm{Zn}}+X_{\mathrm{Mn}}=1, \\
Y_{\mathrm{Co}}+Y_{\mathrm{Zn}}+Y_{\mathrm{Mn}}=1 .
\end{gathered}
$$

In the present study, we determine total amounts of $\mathrm{Co}, \mathrm{Zn}$, and $\mathrm{Mn}$ by the inductively coupled plasma atomic emission spectroscopy (ICP-AES) analysis, which gives another three constraint equations:

$$
12 X_{M}+8 Y_{M}=N_{M}(M=\mathrm{Co}, \mathrm{Zn}, \text { or } \mathrm{Mn}),
$$

where $N_{\mathrm{M}}$ is the total amount of the element $M$ in a unit cell.

By using Eqs. (3)- (8), we obtain two equations,

$$
X_{\mathrm{Mn}}=A-B X_{\mathrm{Co}}
$$

and

$$
X_{\mathrm{Zn}}=1-A+(B-1) X_{\mathrm{Co}}
$$

where

$$
\begin{aligned}
A= & \frac{1}{\left(1+\frac{3}{2} P\right)\left(b_{\mathrm{Mn}}-b_{\mathrm{Zn}}\right)} \\
& \times\left[\frac{P}{8}\left\{\left(8-N_{\mathrm{Co}}-N_{\mathrm{Mn}}\right) b_{\mathrm{Zn}}+N_{\mathrm{Co}} b_{\mathrm{Co}}+N_{\mathrm{Mn}} b_{\mathrm{Zn}}\right\}-b_{\mathrm{Zn}}\right], \\
& B=\frac{b_{\mathrm{Co}}-b_{\mathrm{Zn}}}{b_{\mathrm{Mn}}-b_{\mathrm{Zn}}} .
\end{aligned}
$$

In the case of $\mathrm{Co}_{10} \mathrm{Zn}_{10}$, we can further reduce the number of equations by substituting $X_{\mathrm{Mn}}=0$ into Eq. (9), and then the problem becomes mathematically solvable. In fact, a previous neutron diffraction study on $\mathrm{Co}_{10} \mathrm{Zn}_{10}$ revealed that the $8 \mathrm{c}$ sites are fully occupied by $\mathrm{Co}$ and that $\mathrm{Zn}$ and the remaining Co are in the $12 d$ sites [21,22], as illustrated in Fig. 2(a). This can be interpreted to mean that Co and Zn prefer the $8 c$ and $12 d$ sites, respectively. In the present study, we assume that this tendency also holds in the Mn-substituted samples. Then the problem is now to determine fractions of $\mathrm{Mn}$ in the $8 c$ and $12 d$ sites. For instance, if $\mathrm{Mn}$ would have the strongest site preference for the $12 d$ sites, the rest of the $12 d$ sites would be filled by $\mathrm{Zn}$, and then the $8 c$ sites would be occupied by Co and the rest of $\mathrm{Zn}$, as shown in Fig. 2(b). Another possibility is that $\mathrm{Mn}$ would have the strongest site preference for the $8 \mathrm{c}$ sites, as shown in Fig. 2(c). Actual site occupancies could be something between these two extreme cases [see Fig. 2(d)].

Note that all the samples exhibit the paramagnetic phase at $300 \mathrm{~K}$. We thus consider that the observed intensities contain only the nuclear scattering.

\section{EXPERIMENTAL DETAILS}

Polycrystalline CZM samples with the nominal compositions of $x=4,5,6,7,8,10,12$, and 16 were synthesized from pure Co, $\mathrm{Zn}$, and Mn metals, as described in Ref [19]. Each sample was ground into powder form and loaded in a cylindrical vanadium cell. We also introduced standard $\mathrm{Si}$ powder (SRM 640e, NIST) in each cell as a reference. The amount of Si is approximately $10 \%$ of each CZM sample

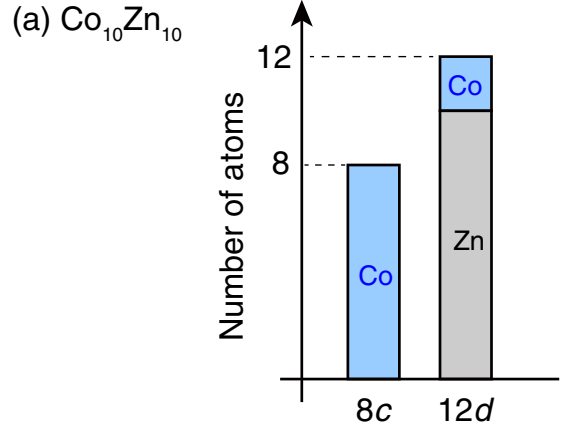

$$
\mathrm{Co}_{10-\times / 2} \mathrm{Zn}_{10-\times / 2} \mathrm{Mn}_{\mathrm{x}} \text { (possible models) }
$$

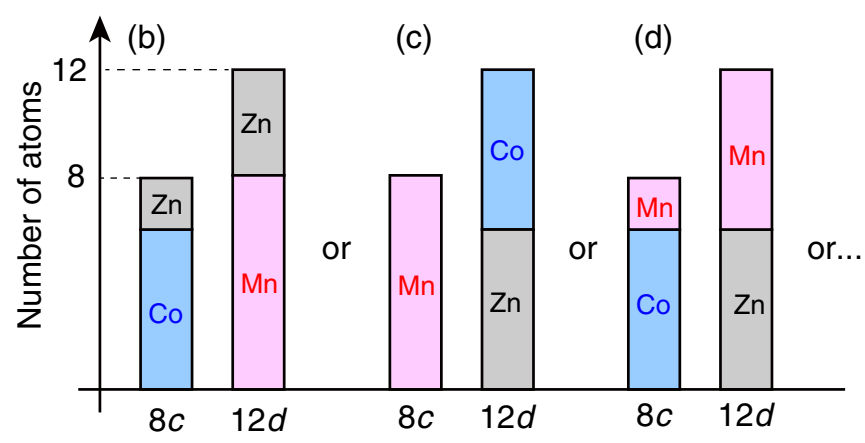

FIG. 2. (a) Site occupancies of $\mathrm{Co}$ and $\mathrm{Zn}$ in $\mathrm{Co}_{10} \mathrm{Zn}_{10}$ reported in Refs. [21,22]. (b)-(d) Possible site occupancies of Co, Zn, and Mn in CZM.

by weight. Neutron diffraction measurements were performed at room temperature using the time-of-flight-type neutron powder diffractometer SPICA [23] on BL09 at the Materials and Life Science Experimental Facility (MLF) in the Japan Proton Accelerator Research Complex (J-PARC). Diffracted neutrons were counted by ${ }^{3} \mathrm{He}$-gas position-sensitive detectors installed in the backscattering detector banks, whose horizontal scattering angle coverage is from $150^{\circ}$ to $175^{\circ}$. Observed diffraction patterns were analyzed by Z-RIETVELD software $[24,25]$. We also measured an XRD pattern of the mixture of the CZM with the $x=10$ sample and the standard Si powder using an x-ray diffractometer (Rigaku RINT TTR-III) with a $\mathrm{Cu} K \alpha$ radiation source.

ICP-AES analyses were performed for $x=4,6,8,12$, and 16 samples by Hitachi Power Solutions Co., Ltd., Japan. The results are summarized in Table I. The nominal and actual compositions agree quite well with each other. We thus fix $N_{\mathrm{Co}}, N_{\mathrm{Zn}}$, and $N_{\mathrm{Mn}}$ in Eqs. (8) and (11) to the nominal values.

TABLE I. Results of the ICP-AES analysis of $\mathrm{Co}_{10-\frac{x}{2}} \mathrm{Zn}_{10-\frac{x}{2}} \mathrm{Mn}_{x}$ samples.

\begin{tabular}{lccc}
\hline \hline Nominal composition & Co $(\%)$ & $\mathrm{Zn}(\%)$ & $\mathrm{Mn}(\%)$ \\
\hline $\mathrm{Co}_{8} \mathrm{Zn}_{8} \mathrm{Mn}_{4}$ & $40.0 \pm 0.8$ & $40.0 \pm 0.8$ & $20.0 \pm 0.4$ \\
$\mathrm{Co}_{7} \mathrm{Zn}_{7} \mathrm{Mn}_{6}$ & $34.9 \pm 0.7$ & $34.7 \pm 0.7$ & $30.4 \pm 0.6$ \\
$\mathrm{Co}_{6} \mathrm{Zn}_{6} \mathrm{Mn}_{8}$ & $29.5 \pm 0.6$ & $29.7 \pm 0.6$ & $40.8 \pm 0.8$ \\
$\mathrm{Co}_{4} \mathrm{Zn}_{4} \mathrm{Mn}_{12}$ & $19.6 \pm 0.4$ & $19.9 \pm 0.4$ & $60.6 \pm 1.2$ \\
$\mathrm{Co}_{2} \mathrm{Zn}_{2} \mathrm{Mn}_{16}$ & $10.0 \pm 0.2$ & $10.3 \pm 0.2$ & $79.7 \pm 1.6$ \\
\hline \hline
\end{tabular}



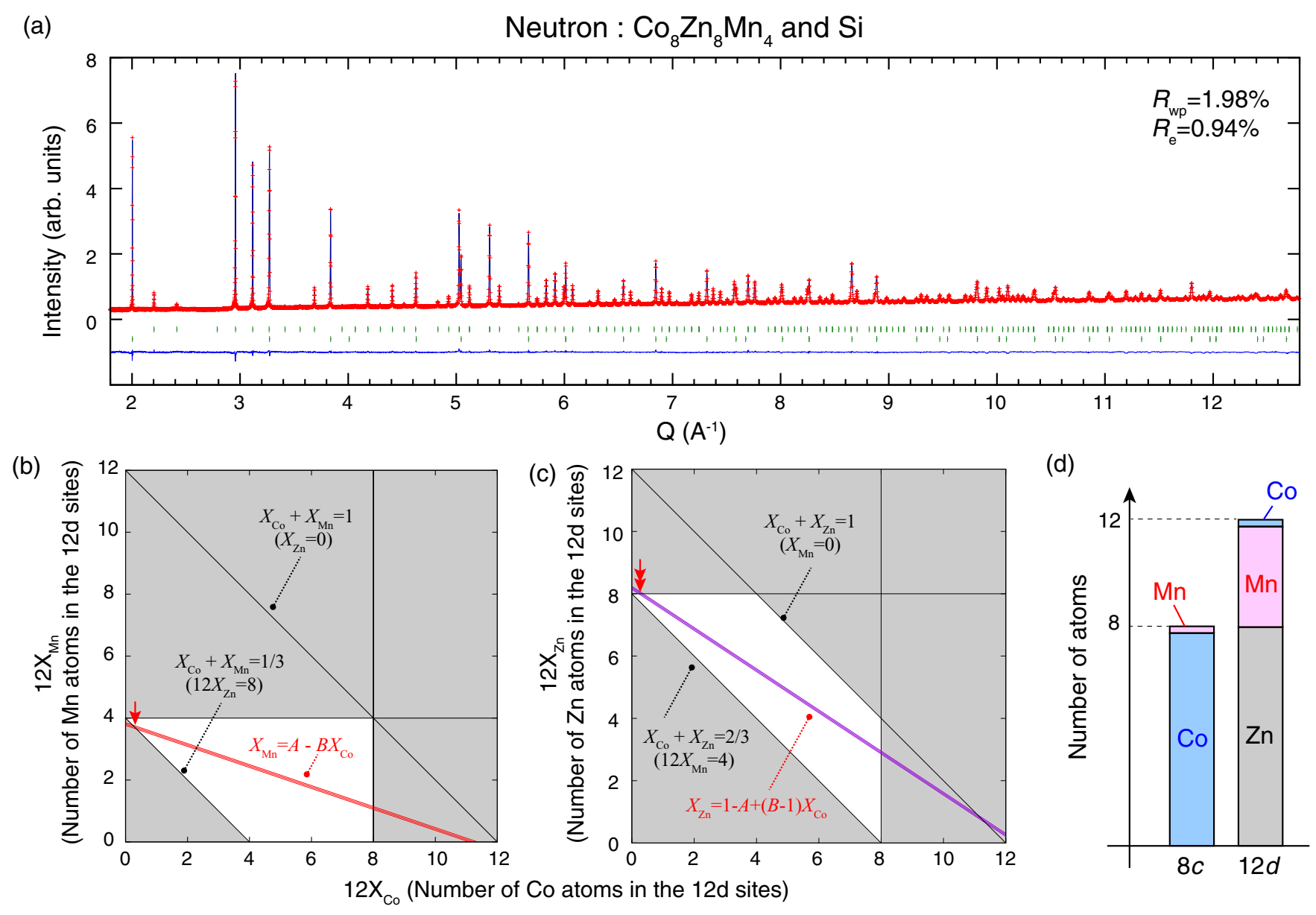

FIG. 3. (a) The result of the Rietveld refinement of the CZM $(x=4)$ sample and Si. The first and second lines of the vertical bars indicate the peak positions for the CZM $(x=4)$ sample and Si, respectively. Possible parameters in the (b) $X_{\mathrm{Co}}-X_{\mathrm{Mn}}$ and (c) $X_{\mathrm{Co}}-X_{\mathrm{Zn}}$ planes (see the text for details). The widths of the solid lines corresponding to Eqs. (9) and (10) represent errors of $X_{\mathrm{Mn}}$ and $X_{\mathrm{Zn}}$ calculated from one standard deviation of $P$ in the Rietveld analysis. (d) The site occupancies of Co, $\mathrm{Zn}$, and $\mathrm{Mn}$ in the $x=4$ sample determined by the present study.

\section{RESULTS AND DISCUSSION}

\section{A. $\mathrm{Co}_{8} \mathrm{Zn}_{8} \mathrm{Mn}_{4}$}

We first discuss the result of the $x=4$ sample as an example to explain how we determine the site occupancies. Figure 3(a) shows the neutron diffraction profile of the $x=4$ sample. All the peaks are indexed by using the known crystal structures of $\mathrm{Co}_{8} \mathrm{Zn}_{8} \mathrm{Mn}_{4}$ and $\mathrm{Si}$. We performed Rietveld analysis of this profile and determined $P$ to be 1.19(4). By using this value, we plot Eqs. (9) and (10) on the $X_{\mathrm{Co}}-X_{\mathrm{Mn}}$ and $X_{\mathrm{Co}}-X_{\mathrm{Zn}}$ planes, as shown in Figs. 3(b) and 3(c), respectively. Because of the constraints of Eqs. (6)-(8) and the fact that $X_{M}$ and $Y_{M}$ cannot be negative, the parameters in the shaded areas are excluded.

From Fig. 3(b) and the equations therein, we determine that $12 X_{\mathrm{Mn}}$ takes the maximum value of 3.71(7) at the crossing point indicated by a red arrow. In this case, most of the Co atoms exist in the $8 c$ sites. This point also corresponds to another crossing point indicated by a double arrow in Fig. 3(c), where all the $\mathrm{Zn}$ atoms are accommodated in the $12 d$ sites. Here, we note that both Eqs. (9) and (10) have negative slopes with respect to $X_{\mathrm{Co}}$. This means that if $X_{\mathrm{Mn}}$ were to decrease, $\mathrm{Zn}$ in the $12 d$ sites and the Co atoms in the $8 c$ sites should be interchanged. In an extreme case when
$X_{\mathrm{Mn}}$ takes the minimum value, all the Co atoms would be accommodated in the $12 d$ sites, and the $8 c$ sites would be filled with $\mathrm{Zn}$ and $\mathrm{Mn}$. Taking into account the previous study on $\mathrm{Co}_{10} \mathrm{Zn}_{10}[21,22]$ reporting that $\mathrm{Co}$ and $\mathrm{Zn}$ prefer the $8 c$ and $12 d$ sites, respectively, we suggest that $12 X_{\mathrm{Mn}}$ takes the maximum value. Then all the $\mathrm{Zn}$ atoms should exist in the $12 d$ sites, as shown in Fig. 3(d). It is noteworthy here that the upper limit of $12 X_{\mathrm{Mn}}$ cannot reach the total amount of $\mathrm{Mn}$, that is, $N_{\mathrm{Mn}}=4$, revealing that a small amount of Mn should exist in the $8 c$ site. As a result, some Co atoms are accommodated in the $12 d$ sites, while most Co atoms are in the $8 c$ sites.

\section{B. $x$ dependence of the site occupancies and the average scattering lengths}

We analyzed the data of the $x=5,6,7,8,12$, and 16 samples in the same manner as that for the $x=4$ sample. The results of the Rietveld refinements are found in the Appendix. Figure 4 summarizes $N_{\mathrm{Mn}}$ and the upper limit of $12 X_{\mathrm{Mn}}$ as functions of $x$. Here, the upper limit has been experimentally determined without assuming the site preferences of Co and $\mathrm{Zn}$. We found that $12 X_{\mathrm{Mn}}$ never exceeds $12-N_{\mathrm{Zn}}$, implying that there is always room for all the $\mathrm{Zn}$ to be accommodated in 


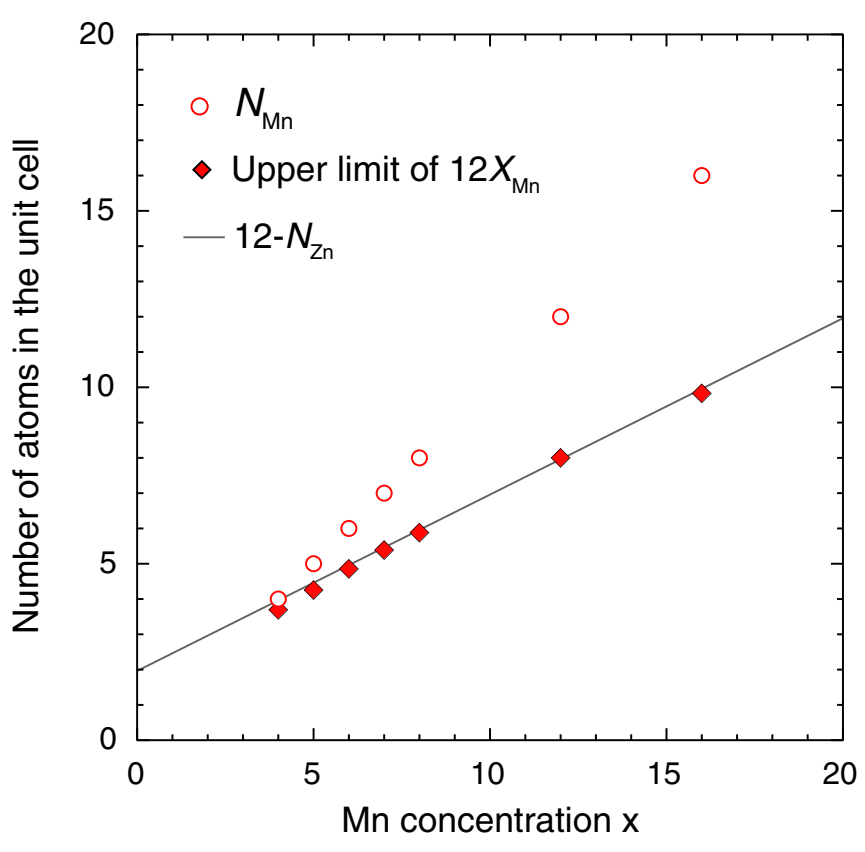

FIG. 4. $x$ dependence of $N_{\mathrm{Mn}}$ and the upper limit of $12 X_{\mathrm{Mn}}$ determined by the present study. The gray solid line shows $12-N_{\mathrm{Zn}}$.

the $12 d$ sites. We thus conclude that all the $\mathrm{Zn}$ atoms are in the $12 d$ sites regardless of the $\mathrm{Mn}$ concentration. On the basis of these experimental results and considerations, we determine the site occupancies of $\mathrm{Co}, \mathrm{Zn}$, and $\mathrm{Mn}$ for all the samples, as shown in Figs. 1(b) and 1(c). Note that there were several previous studies on crystal structures of CZM compounds with relatively low Mn concentration [21,26] which also reported that the $\mathrm{Mn}$ atoms mainly prefer the $12 d$ sites. The present results show good agreement with them in the low- $x$ region and reveal the site occupancies in the whole range of $x$.

Having established the site occupancies, the average scattering lengths are calculated as functions of $x$, as shown in Fig. 5(a). Because Mn has a negative scattering length, both $\left\langle b_{8 c}\right\rangle$ and $\left\langle b_{12 d}\right\rangle$ are monotonically reduced with increasing $x$. Interestingly, both of them become nearly zero at around $x=10$. In fact, we found that the Bragg reflections from the CZM sample with $x=10$ were hardly visible in neutron diffraction, while they were clearly observed in XRD, as shown in Figs. 5(b) and 5(c), respectively. This is another verification of our model.

We note here that the absolute values of $\left\langle b_{8 c}\right\rangle$ and $\left\langle b_{12 d}\right\rangle$ can also be determined by comparing the intensities of Bragg reflections of the CZM samples with those of Si. We confirmed that the data shown in Fig. 5(a) show good agreement with the absolute scattering lengths determined by the comparison in intensity with $\mathrm{Si}$.

\section{Correlation between the site occupancies and the spin-glass transition}

In the $x=0$ sample, the $8 c$ sites are fully occupied by magnetic $\mathrm{Co}$, while two thirds of the $12 d$ sites are occupied by nonmagnetic $\mathrm{Zn}[21,22]$, as we mentioned in previous sections. Therefore, in the low- $x$ range, the magnetic
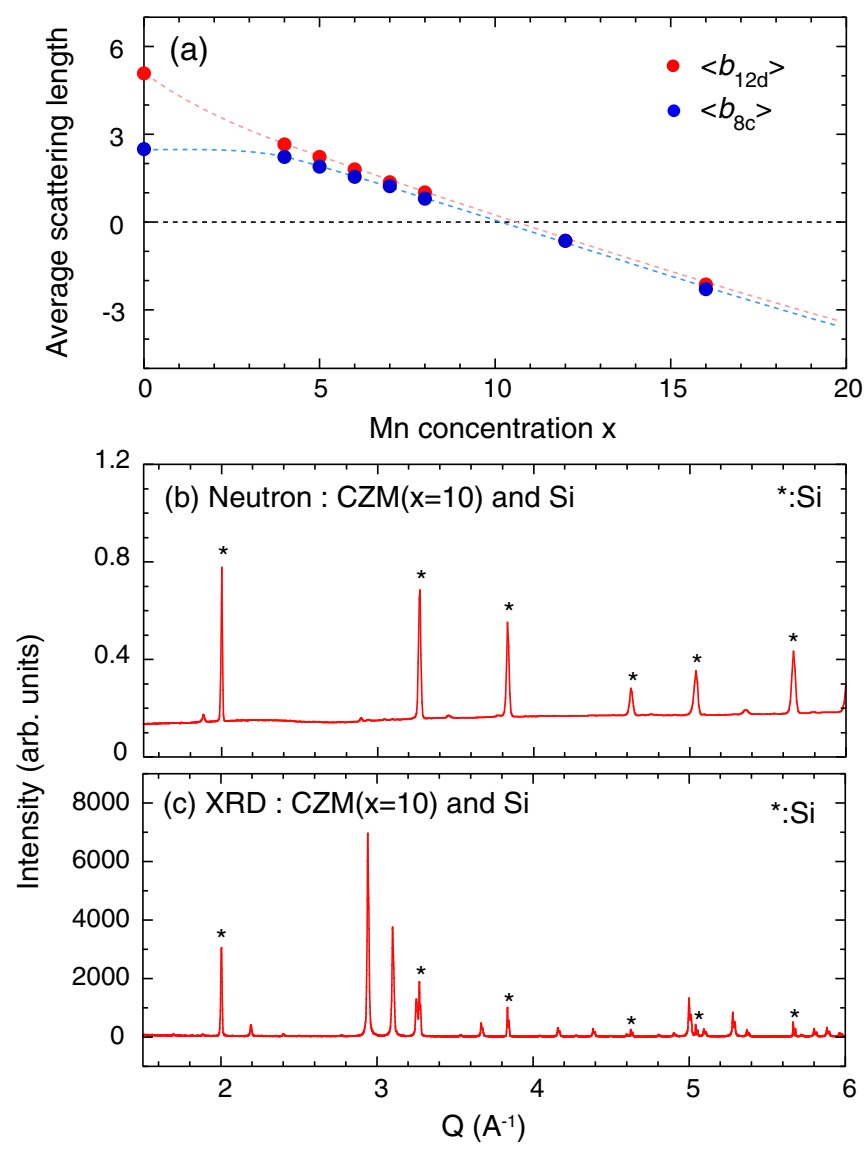

FIG. 5. (a) Averaged coherent scattering lengths calculated from the site occupancies shown in Figs. 1(b) and 1(c). Dotted lines are guides to the eyes. (b) The neutron diffraction and (c) XRD profiles of the mixture of the CZM with the $x=10$ sample and the standard $\mathrm{Si}$ powder. The asterisks show the Bragg peaks from $\mathrm{Si}$.

properties of the system are supposed to be governed by the $8 c$ sites. The present study has revealed that the evolutions of the spin-glass transition temperature and the number of Mn atoms in the $8 c$ sites nearly coincide with each other in the range of $3 \leqslant x \leqslant 7$, as shown in Figs. 1(a) and 1(b). Considering that a direct exchange interaction between $\mathrm{Mn}$ atoms is likely antiferromagnetic, we suggest that the random distribution of the ferromagnetic and antiferromagnetic interactions between the $8 c$ sites induces magnetic disorder, which should reduce the magnitude of $J$ on average, and can also be a source of the spin-glass state.

It was also reported that the end compound $\beta-\mathrm{Mn}(x=20)$ exhibits a spin-liquid state owing to strong magnetic frustration $[27,28]$. The connections between neighboring $12 d$ sites form three-dimensional corner-sharing triangles, the socalled hyperkagome lattice. The antiferromagnetic Mn-Mn interactions on the triangular network may lead to geometrical spin frustration, which could also contribute to the emergence of the spin-glass state. Actually, a recent study on CZM with relatively low Mn concentrations (up to $x=6$ ) [26] reported that the magnetic moments in the $8 c$ and $12 d$ sublattices show different temperature evolutions; the net magnetic moment in the $12 d$ sites is significantly reduced at low temperature, implying that the $\mathrm{Mn}$ moments on the $12 d$ sites are 


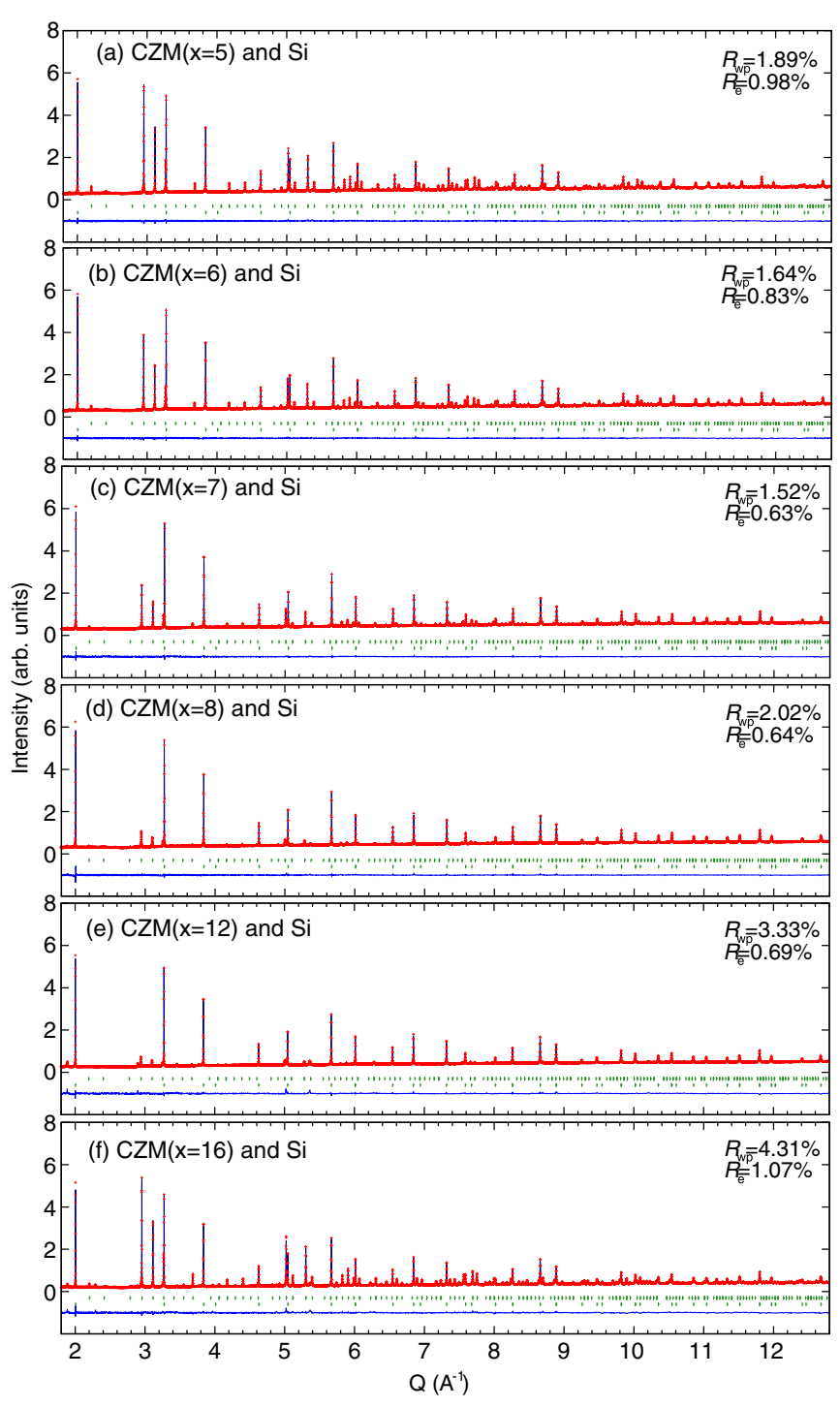

FIG. 6. The results of the Rietveld refinements on the neutron diffraction profiles of the CZM samples with (a) $x=5$, (b) 6 , (c) 7 , (d) 8 , (e) 12, and (f) 16 . The first and second lines of the vertical bars indicate the peak positions for the CZM samples and $\mathrm{Si}$, respectively.

disordered at low temperatures. However, the present results revealed that the frustrated $12 d$ sublattice is mostly occupied by nonmagnetic $\mathrm{Zn}$ in the low- $x$ region and that the reentrant spin-glass state becomes pronounced above $x=4$, where the Mn atoms start occupying the $8 c$ sites. These results imply the importance of magnetic disorder in the $8 c$ sites for the spin-glass state.

\section{SUMMARY}

We have studied the crystal structures of skyrmion-host $\mathrm{Co}_{10-\frac{x}{2}} \mathrm{Zn}_{10-\frac{x}{2}} \mathrm{Mn}_{x}$ with $x=4,5,6,7,8,10,12$, and 16 by means of neutron powder diffraction and the ICP-AES analysis in order to investigate the microscopic mechanism of the Mn substitution effect. We have experimentally determined the upper and lower limits of the Mn fraction for the two independent crystallographic sites, namely, the $8 c$ and $12 d$
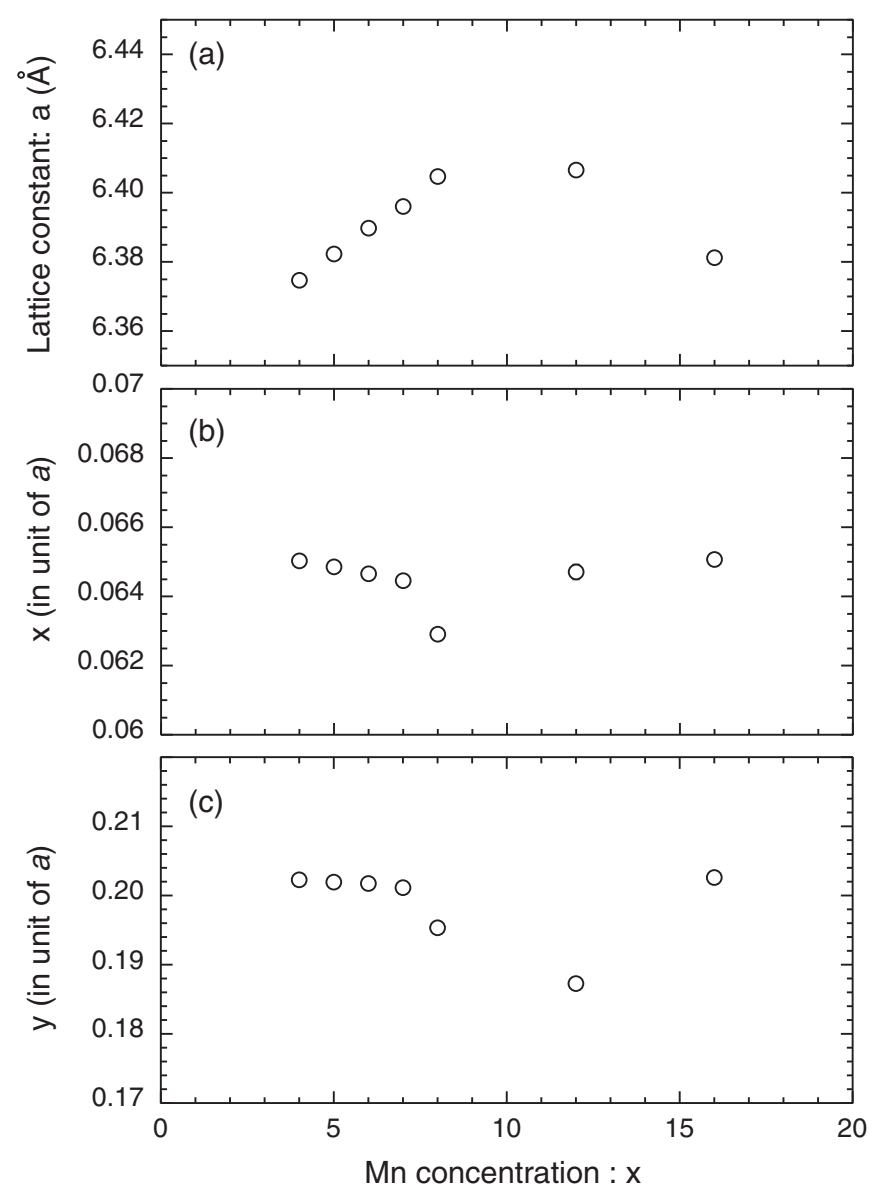

FIG. 7. The $x$ dependence of (a) the lattice constant $a$, (b) the fractional coordinate $x$ for the $8 c$ site at $(x, x, x)$, and (c) $y$ for the $12 d$ site at $\left(\frac{1}{8}, y, \frac{1}{4}+y\right)$.

sites. By assuming that the site preferences of $\mathrm{Co}$ and $\mathrm{Zn}$ reported in $\mathrm{Co}_{10} \mathrm{Zn}_{10}$ also hold in the Mn-doped samples, we conclude that Mn prefers the $12 d$ sites but cannot be fully accommodated because $\mathrm{Zn}$ has a stronger site preference for the $12 d$ sites. We have also found a correlation between the amount of $\mathrm{Mn}$ in the $8 c$ sites and the spin-glass transition temperature, implying that the antiferromagnetic Mn-Mn correlation in the $8 c$ sublattice should introduce some randomness in exchange interactions, which accounts for the reduction of the net ferromagnetic interaction and the emergence of the spinglass state. The present study will contribute to understanding of the microscopic origin of the robust metastable skyrmion and disordered skyrmion states discovered in the CZM family [17-19].

\section{ACKNOWLEDGMENTS}

This work was partly supported by JSPS KAKENHI (Grants No. 24224009, and No. 17K18355). The neutron scattering experiment at MLF of J-PARC was approved by the Neutron Scattering Program Advisory Committee of IMSS, KEK (Proposal No. 2014S10). We thank E. Canévet, D. V. Cheptiakov, and V. Pomjakushin for their support with preliminary powder neutron diffraction measurements 
performed at the Swiss Spallation Neutron Source (SINQ), Paul Scherrer Institute (PSI), Switzerland. We acknowledge financial support from the Swiss National Science Foundation (SNF) Project Grants No. 153451 and No. 166298 and the SNSF Sinergia network NanoSkyrmionics (Grant No. CRSII5_171003). The image of the crystal structure shown in Fig. 1(a) was depicted using the software VESTA [29] developed by K. Momma.

\section{APPENDIX: RESULTS OF THE RIETVELD REFINEMENTS}

Figures 6(a)-6(f) show the results of the Rietveld analysis of mixtures of the CZM ( $x=5,6,7,8,12$, and 16) and standard silicon samples. These neutron diffraction profiles were measured using the SPICA diffractometer in MLF of J-PARC. The $x$ dependence of the lattice constant and the fractional coordinates are summarized in Figs. 7(a)-7(c).
[1] S. Mühlbauer, B. Binz, F. Jonietz, C. Pfleiderer, A. Rosch, A. Neubauer, R. Georgii, and P. Böni, Science 323, 915 (2009).

[2] X. Z. Yu, Y. Onose, N. Kanazawa, J. H. Park, J. H. Han, Y. Matsui, N. Nagaosa, and Y. Tokura, Nature (London) 465, 901 (2010).

[3] N. Nagaosa and Y. Tokura, Nat. Nanotechnol. 8, 899 (2013).

[4] W. Münzer, A. Neubauer, T. Adams, S. Mühlbauer, C. Franz, F. Jonietz, R. Georgii, P. Böni, B. Pedersen, M. Schmidt, A. Rosch, and C. Pfleiderer, Phys. Rev. B 81, 041203(R) (2010).

[5] X. Z. Yu, N. Kanazawa, Y. Onose, K. Kimoto, W. Z. Zhang, S. Ishiwata, Y. Matsui, and Y. Tokura, Nat. Mater. 10, 106 (2011).

[6] S. Seki, X. Z. Yu, S. Ishiwata, and Y. Tokura, Science 336, 198 (2012).

[7] A. Fert, V. Cros, and J. Sampaio, Nat. Nanotechnol. 8, 152 (2013).

[8] W. Koshibae, Y. Kaneko, J. Iwasaki, M. Kawasaki, Y. Tokura, and N. Nagaosa, Jpn. J. Appl. Phys. 54, 053001 (2015).

[9] K. Litzius, I. Lemesh, B. Krüger, P. Bassirian, L. Caretta, K. Richter, F. Büttner, K. Sato, O. A. Tretiakov, J. Förster et al., Nat. Phys. 13, 170 (2016).

[10] X. Zhang, M. Ezawa, and Y. Zhou, Sci. Rep. 5, 9400 (2015).

[11] K. Shibata, X. Z. Yu, T. Hara, D. Morikawa, N. Kanazawa, K. Kimoto, S. Ishiwata, Y. Matsui, and Y. Tokura, Nat. Nanotechnol. 8, 723 (2013).

[12] T. Koretsune, N. Nagaosa, and R. Arita, Sci. Rep. 5, 13302 (2015).

[13] S. V. Grigoriev, S.-A. Siegfried, E. V. Altynbayev, N. M. Potapova, V. Dyadkin, E. V. Moskvin, D. Menzel, A. Heinemann, S. N. Axenov, L. N. Fomicheva et al., Phys. Rev. B 90, 174414 (2014).

[14] L. J. Bannenberg, K. Kakurai, F. Qian, E. Leliévre-Berna, C. D. Dewhurst, Y. Onose, Y. Endoh, Y. Tokura, and C. Pappas, Phys. Rev. B 94, 104406 (2016).

[15] K. Karube, K. Shibata, J. S. White, T. Koretsune, X. Z. Yu, Y. Tokunaga, H. M. Rønnow, R. Arita, T. Arima, Y. Tokura et al., Phys. Rev. B 98, 155120 (2018).
[16] Y. Tokunaga, X. Z. Yu, J. S. White, H. M. Ronnow, D. Morikawa, Y. Taguchi, and Y. Tokura, Nat. Commun. 6, 7638 (2015).

[17] K. Karube, J. S. White, N. Reynolds, J. L. Gavilano, H. Oike, A. Kikkawa, F. Kagawa, Y. Tokunaga, H. M. Rønnow, Y. Tokura et al., Nat. Mater. 15, 1237 (2016).

[18] K. Karube, J. S. White, D. Morikawa, M. Bartkowiak, A. Kikkawa, Y. Tokunaga, T. Arima, H. M. Rønnow, Y. Tokura, and Y. Taguchi, Phys. Rev. Mater. 1, 074405 (2017).

[19] K. Karube, J. S. White, D. Morikawa, C. D. Dewhurst, R. Cubitt, A. Kikkawa, X. Z. Yu, Y. Tokunaga, T. Arima, H. M. Rønnow et al., Sci. Adv. 14, eaar7043 (2018).

[20] K. Karube, J. S. White et al. (unpublished).

[21] T. Hori, H. Shiraishi, and Y. Ishii, J. Magn. Magn. Mater. 310, 1820 (2006).

[22] W. Xie, S. Thimmaiah, J. Lamsal, J. Liu, T. W. Heitmann, D. Quirinale, A. I. Goldman, V. Pecharsky, and G. J. Miller, Inorg. Chem. 52, 9399 (2013).

[23] M. Yonemura, K. Mori, T. Kamiyama, T. Fukunaga, S. Torii, M. Nagao, Y. Ishikawa, Y. Onodera, and D. Adipranoto, J. Phys.: Conf. Ser. 502, 012053 (2014).

[24] R. Oishi, M. Yonemura, Y. Nishimaki, S. Torii, A. Hoshikawa, T. Ishigaki, T. Morishima, K. Mori, and T. Kamiyama, Nucl. Instrum. Methods Phys. Res., Sect. A 600, 94 (2009).

[25] R. Oishi-Tomiyasu, M. Yonemura, T. Morishima, A. Hoshikawa, S. Torii, T. Ishigaki, and T. Kamiyama, J. Appl. Crystallogr. 45, 299 (2012).

[26] J. D. Bocarsly, C. Heikes, C. M. Brown, S. D. Wilson, and R. Seshadri, Phys. Rev. Mater. 3, 014402 (2019).

[27] J. A. M. Paddison, J. R. Stewart, P. Manuel, P. Courtois, G. J. McIntyre, B. D. Rainford, and A. L. Goodwin, Phys. Rev. Lett. 110, 267207 (2013).

[28] H. Nakamura, K. Yoshimoto, M. Shiga, M. Nishi, and K. Kakurai, J. Phys.: Condens. Matter 9, 4701 (1997).

[29] K. Momma and F. Izumi, J. Appl. Crystallogr. 44, 1272 (2011). 